\title{
ẢNH HƯỞNG HỖ TRỢ CỦA CÁC ION IODUA ĐẾN SỰ ỨC CHẾ ĂN MÒN THÉP CARBON BỎ̉ HEXAMETHYLENETETRAMINE TRONG NƯỚC VÀ NHŨ TƯƠNG "CONDENSATE/NƯỚC"
}

\author{
Vũ Đình Huy ${ }^{*}$, Lưu Hoàng Tâm, Lê Văn Thuận, Thái Nhi Hiệp, Phan Thanh Bình
}

Trường Đại học Bách khoa, 268 Lý Thuờng Kiệt, TP. Hồ Chí Minh

"Email: huy_vudinh@yahoo.com

Đến Tòa soạn: 12/6/2013; Chấp nhận đăng: 31/8/2013

\section{TÓM TẮT}

Tác dụng hỗ trợ của các ion iodua $\left(\mathrm{I}^{-}\right)$đến sự ức chế ăn mòn thép carbon trong nước và các nhũ tương "condensate/nước" bởi hexametylentetramine $\left(\mathrm{C}_{6} \mathrm{H}_{12} \mathrm{~N}_{4}\right)$, đã được nghiên cứu bằng các phương pháp khối lượng, phân cực thế động, phân cực tuyến tính và trở kháng điện hóa. Hexametylentetramine ức chể sự ăn mòn thép carbon ở những nồng độ thấp. Hiệu quả ức chế ăn mòn thép carbon của hexametylentetramine tăng với sự tăng nồng độ của ion iodua và với sự giảm hàm lượng nước trong các nhũ tương “condensate/ nước". Hiệu quả ức chế ăn mòn thép carbon bởi hốn hợp "Hexametylentetramine $(2 \mathrm{~g} / \mathrm{l})+\mathrm{KI}(0 ; 0,5 ; 1 ; 2 \mathrm{~g} / \mathrm{l})$ " được tăng đáng kể trong các dung dịch nước đã loại bỏ khí oxy. Hiệu quả cao nhất của sự ức chế ăn mòn thép carbon bởi hỗn hợp "Hexametylentetramine $(2 \mathrm{~g} / \mathrm{l})+\mathrm{KI}(0 ; 0,5 ; 1 ; 2 \mathrm{~g} / \mathrm{l})$ " quan sát thấy ở nhiệt độ $100{ }^{\circ} \mathrm{C}$ trong khoảng nhiệt độ từ $30^{\circ} \mathrm{C}$ đến $140{ }^{\circ} \mathrm{C}$. Cơ chế ức chế ăn mòn thép carbon bởi hỗn hợp "Hexametylentetramine + KI" được giải thích là do có sự tạo thành lớp hâp phụ kép trên bề mặt thép.

Tù khóa: ion iodua, hexametylentetramine, ức chế ăn mòn, thép carbon, nước, nhũ tương "condensate/ nước".

\section{MỞ ĐẦU}

Condensate là hỗn hợp hydrocarbon lỏng, mạch thẳng, tỉ trọng thấp, được dùng để sản xuất xăng, dung môi công nghiệp và làm nguyên liệu cho tổ hợp hóa dầu. Một lượng nhỏ nước chứa các axit và khí oxy nhiễm vào condensate có thể gia tốc sự ăn mòn kim loại trong quá trình xử lí condensate ở nhiệt độ cao. Biện pháp thích hợp nhất nhằm kìm hãm quá trình ăn mòn kim loại nói trên là sử dụng chất ức chế.

Hexamethylenetetramine $\left(\mathrm{C}_{6} \mathrm{H}_{12} \mathrm{~N}_{4}\right)$ là chất ức chế ăn mòn thép không gây ô nhiễm môi trường. Nó ức chế ăn mòn thép carbon trong dung dịch tẩy gỉ theo tiêu chuẩn Mỹ ASTM G1-90 [1], ức chế ăn mòn thép mềm trong axit clohydric $\mathrm{HCl}$ [2], ức chế ăn mòn đồng trong môi trường bromua [3]. 
Các ion iodua $\left(\mathrm{I}^{-}\right)$chỉ có tính chất hỗ trợ, tăng thêm khả năng ức chế ăn mòn thép mềm bởi những chất ức chế chính trong các dung dịch axit [4 - 14].

Trong nghiên cứu này, chúng tôi khảo sát ảnh hưởng hỗ trợ của Kali iodua đến sự ức chế ăn mòn thép carbon bởi Hexamethylenetetramine (viết tắt là Hexa) trong nước và các nhũ tương "condensate/nước" không hoặc có khí oxy hòa tan, trên khoảng nhiệt độ từ $30^{\circ} \mathrm{C}$ đến $140{ }^{\circ} \mathrm{C}$.

\section{PHƯƠNG PHÁP NGHIÊN CÚU}

\section{1. Đối tượng nghiên cứu}

Thép cacbon N80, được cắt từ đường ống dẫn condensate. Phân tích bằng phổ kế phát xạ DIA 2000SE cho thành phần của thép như sau (\% khối lượng):

C $(0,253), \mathrm{Si}(0,334), \mathrm{Mn}(1,33), \mathrm{P}(0,023), \mathrm{S}(0,002), \mathrm{Cr}(0,204), \mathrm{Mo}(0,002), \mathrm{Ni}(0,031), \mathrm{Al}$ $(0,035), \mathrm{Co}(0,004), \mathrm{Cu}(0,015), \mathrm{Nb}(0,002), \mathrm{Ti}(0,008), \mathrm{V}(<0,001), \mathrm{W}(<0,01), \mathrm{Pb}(<0,003), \mathrm{Sn}$ $(<0,001)$, As $(<0,004), \mathrm{Zr}(0,001), \mathrm{Ca}(0,002), \mathrm{Ce}(0,006), \mathrm{Ta}(0,022), \mathrm{B}(<0,0000001), \mathrm{Zn}$ $(0,008), \mathrm{La}(<0,001), \mathrm{Fe}(<97,7)$.

* Thành phần hóa học của condensate (\% khối lượng ):

Propane $(0,447)$; i-butane $(3,252)$; n-butane $(7,245) ; 2,2$-dimethylpropane $(0,077)$; i-pentane (9,467); n-pentane (7,412); 2,2-dimethylbutane $(0,326)$; benzene $(1,936)$; toluen $(3,843)$.

Nước có lẫn condensate được lấy tại đáy bồn chứa của một nhà máy chế biến condensate.

* Thành phần hóa học của nuóc: Độ dẫn điện (13510 $\mu \mathrm{S} / \mathrm{cm})$; Độ pH (4,77); Độ cứng (255 ppm); Độ kiềm tổng (1248 ppm); $\mathrm{Cl}^{-}(84 \mathrm{mg} / \mathrm{l}) ; \mathrm{SiO}_{2}(195,57 \mathrm{mg} / \mathrm{l}) ; \mathrm{PO}_{4}^{3-}(0,45 \mathrm{mg} / \mathrm{l}) ;$ Tổng $\mathrm{Fe}^{2+/ 3+}(0,21 \mathrm{mg} / \mathrm{l}) ; \mathrm{Cu}^{2+}(0,04 \mathrm{mg} / \mathrm{l})$; lượng vi khuẩn (<10 $10^{4}$ tế bào/ml).

Các hóa chất thí nghiệm: Hexa, KI, Natri sulfit $\left(\mathrm{Na}_{2} \mathrm{SO}_{3}\right)$ là loại tinh khiết $(99 \%)$.

\subsection{Chuẩn bị mẫu thí nghiệm}

\subsubsection{Thí nghiệm ở các nhiệt độ khác nhau trong nước đã loại khí oxy, bằng phuơng pháp khối luọng}

Mỗi thí nghiệm sử dụng $400 \mathrm{ml}$ dung dịch nước trong bình thép không gỉ, kín, dung tích $500 \mathrm{ml}$ và 2 mẫu thép phẳng, kích thước: $50 \times 15 \times 3 \mathrm{~mm}$, khoan 2 lỗ, đường kính lỗ $2 \mathrm{~mm}$. Xử lí mẫu thép trước và sau thí nghiệm theo tiêu chuẩn ASTM G1-90 [1]. Cân mẫu bằng cân XT220A Precisa, có độ chính xác $\pm 0,0001$ g.

Loại khí oxy hòa tan trong nước bằng muối Natri sunfit: $2 \mathrm{Na}_{2} \mathrm{SO}_{3}+\mathrm{O}_{2} \rightarrow 2 \mathrm{Na}_{2} \mathrm{SO}_{4}$

Mỗi thí nghiệm thực hiện 4 giờ ở các nhiệt độ $30^{\circ} \mathrm{C}, 60^{\circ} \mathrm{C}, 100{ }^{\circ} \mathrm{C}, 140{ }^{\circ} \mathrm{C}$, theo tiêu chuẩn ASTM G111-92 [15]. Nồng độ các hóa chất thí nghiệm: Hexa $(2 \mathrm{~g} / \mathrm{l})+\mathrm{KI}(0 ; 0,5 ; 1 ; 2 \mathrm{~g} / \mathrm{l})$.

2.2.2. Thí nghiệm ở nhiệt độ phòng $\left(30^{\circ} \mathrm{C} \pm 1{ }^{\circ} \mathrm{C}\right)$ trong nước và nhũ tuoong "condensate/nuoớc", khuấy liên tục, thông khí, bằng các phuơng pháp khối lượng và điện hóa

- Phương pháp khối lượng: Mẫu thép phẳng, kích thước: $50 \times 15 \times 3$ mm, khoan 2 lỗ, đường kính lỗ $2 \mathrm{~mm}$. Tỉ lệ thể tích "Condensate/ nước" = (0:100); (50:50); (70: 30) và (90:10). giờ.

Khuấy các dung dịch liên tục bằng máy khuấy từ CB302, 200 vòng/phút, trong: 4,8 và 24 
Tính tốc độ ăn mòn thép theo công thức: $\mathrm{V}=\frac{K \times \mathrm{W}}{A \times T \times D}$

trong đó: $\mathrm{V}$ - Tốc độ ăn mòn thép (mm/năm); $\mathrm{K}=8,76 \times 10^{4} ; \mathrm{W}$ - Tổn thất khối lượng thép do ăn mòn $(\mathrm{g}) ; \mathrm{A}$ - Diện tích mẫu $\left(\mathrm{cm}^{2}\right)$; T - Thời gian ngâm mẫu $(\mathrm{h}) ; \mathrm{D}$ - Khối lượng riêng của thép.

Hiệu quả bảo vệ thép Z (\%) tính theo công thức:

$$
\mathrm{Z}(\%)=\frac{V_{0}-v_{1}}{V_{o}} \times 100
$$

ở đây: $\mathrm{V}_{\mathrm{o}}, \mathrm{V}_{1}$ - Tốc độ ăn mòn thép trong dung dịch không và có chất ức chế.

- Phương pháp điện hóa: Sử dụng thiết bị Solatron 1280B để đo các đường cong phân cực anot, catot và tổng trở điện hóa. Bình đo chứa ba điện cực: Điện cực làm việc là thép N80 (đúc trong epoxy, bề mặt thép tiếp xúc với dung dịch có kích thước: $15,15 \mathrm{~mm} \times 14,78 \mathrm{~mm}$ ), điện cực so sánh là điện cực Calomen bão hòa (SCE), điện cực đối là lưới Platin.

Đo đường cong phân cực catot và anot theo tiêu chuẩn ASTM G5- 94 [16] và ASTM G5991 [17]. Quét điện thế từ giá trị $\left(\mathrm{E}_{\mathrm{corr}}-500 \mathrm{mV}\right)$ đến $\left(\mathrm{E}_{\mathrm{corr}}+500 \mathrm{mV}\right)$, với tốc độ quét $5 \mathrm{mV} / \mathrm{s}$. ( $E_{\text {corr }}$ là điện thế ăn mòn thép). Đo điện trở phân cực từ giá trị $\left(E_{\text {corr }}-10 \mathrm{mV}\right)$ đến $\left(\mathrm{E}_{\text {corr }}+10 \mathrm{mV}\right)$.

Mật độ dòng ăn mòn ( $\mathrm{i}_{\text {corr }}$ ), tốc độ ăn mòn $(\mathrm{V})$ được xác định bằng hai phương pháp: ngoại suy Tafel và đo điện trở phân cực tuyến tính.

Đo tổng trở điện hóa theo tiêu chuẩn ASTM G106 - 89 [18], bằng thiết bị Solatron 1280B với phần mềm $\mathrm{Z}$ plot. Phép đo thực hiện ở giá trị điện thế ăn mòn $\left(\mathrm{E}_{\mathrm{corr}}\right)$, tốc độ quét điện thế 1 $\mathrm{mV} / \mathrm{sec}$; biên độ nhiễu loạn sóng hình sin là $10 \mathrm{mV}$ trong khoảng tần số từ $20.000 \mathrm{~Hz}$ đến $0,1 \mathrm{~Hz}$.

\section{KẾT QUẢ VÀ THẢO LUẬN}

\subsection{Kết quả thí nghiệm trong nước đã loại khí oxy ở các nhiệt độ khác nhau}

Bảng 1 cho thấy, sau 4 giờ ngâm mẫu thép trong nước đã loại khí oxy, tốc độ ăn mòn thép bị tăng dần theo chiều tăng của nhiệt độ; nhưng tốc độ ăn mòn thép lại giảm dần theo chiều tăng của nồng độ KI trong hỗn hợp chất ức chế "Hexa 2 g/l + KI )".

Bảng 1. Tốc độ ăn mòn thép trong nước đã loại khí oxy, phụ thuộc vào nhiệt độ và nồng độ của hỗn hợp "Hexa (2 g/l) + KI (0; 0,5;1;2 g/l)".

\begin{tabular}{|c|c|c|c|c|c|}
\hline \multirow{2}{*}{$\begin{array}{c}\text { Chất ức chế } \\
\text { Hexa }(\mathbf{g} / \mathbf{l})\end{array}$} & \multirow{2}{*}{$+\mathbf{K I}(\mathbf{g} / \mathbf{l})$} & \multicolumn{4}{|c|}{ Tốc độ ăn mòn thép $(\mathbf{m m} / \mathbf{n a ̆ m})$} \\
\cline { 3 - 6 } & & $\mathbf{3 0}^{\mathbf{0}} \mathbf{C}$ & $\mathbf{6 0}^{\mathbf{0}} \mathbf{C}$ & $\mathbf{1 0 0}^{\mathbf{0}} \mathbf{C}$ & $\mathbf{1 4 0}^{\mathbf{0}} \mathbf{C}$ \\
\hline 0 & 0 & 0,417 & 1,717 & 3,033 & 3,224 \\
\hline 2 & +0 & 0,335 & 1,205 & 1,353 & 1,637 \\
\hline 2 & $+0,5$ & 0,315 & 1,123 & 1,296 & 1,503 \\
\hline 2 & +1 & 0,213 & 0,748 & 1,034 & 1,291 \\
\hline 2 & +2 & 0,194 & 0,725 & 0,965 & 1,214 \\
\hline
\end{tabular}


Bảng 2 cho thấy, trong nước đã loại khí oxy, hiệu quả bảo vệ thép của Hexa $(2 \mathrm{~g} / \mathrm{l})$ và của "Hexa $(2 \mathrm{~g} / \mathrm{l})+\mathrm{KI}$ " đều đạt giá trị cực đại tại nhiệt độ $100{ }^{\circ} \mathrm{C}$, và tăng dần theo chiều tăng của nồng độ KI.

Bảng 2. Hiệu quả bảo vệ thép bởi hỗn hợp "Hexa (2 g/l) + KI (0;0,5;1;2 g/l)" trong nước đã loại khí oxy, phụ thuộc vào nhiệt độ và nồng độ của KI.

\begin{tabular}{|c|c|c|c|c|c|}
\hline \multirow{2}{*}{$\begin{array}{c}\text { Chất ức chế } \\
\text { Hexa }(\mathbf{g} / \mathbf{l})\end{array}$} & \multirow{2}{*}{$+\mathbf{K I}(\mathbf{g} / \mathbf{l})$} & \multicolumn{4}{|c|}{ Hiệu quả bảo vệ thép $(\boldsymbol{\%})$} \\
\cline { 3 - 6 } & & $\mathbf{3 0}^{\mathbf{0}} \mathbf{C}$ & $\mathbf{6 0}^{\mathbf{0}} \mathbf{C}$ & $\mathbf{1 0 0}^{\mathbf{}} \mathbf{C}$ & $\mathbf{1 4 0}^{\mathbf{0}} \mathbf{C}$ \\
\hline 2 & +0 & $19,70 \%$ & 29,84 & 55,46 & $49,22 \%$ \\
\hline 2 & $+0,5$ & 24,49 & 34,59 & 57,27 & 53,39 \\
\hline 2 & +1 & 48,88 & 56,47 & 65,90 & 59,95 \\
\hline 2 & +2 & 53,51 & 57,74 & 68,19 & 62,34 \\
\hline
\end{tabular}

\subsection{Kết quả thí nghiệm trong nước, nhũ tương “condensate/nước" thông khí, ở nhiệt độ} phòng bằng phương pháp khối lượng

Bảng 3. Tốc độ ăn mòn thép phụ thuộc vào nồng độ của "Hexa $(2 \mathrm{~g} / \mathrm{l})+\mathrm{KI}$ ", vào thời gian ngâm mẫu (h) và hàm lượng nước (\%) trong các nhũ tương “condensate / nước” thông khí, ở nhiệt độ phòng.

\begin{tabular}{|c|c|c|c|c|c|c|}
\hline & \multicolumn{5}{|c|}{ Tốc độ ăn mòn thép (mm/năm) } \\
\hline & & \multirow{2}{*}{$\begin{array}{l}\text { Không có } \\
\text { chất ức chế }\end{array}$} & \multirow{2}{*}{\begin{tabular}{|c|}
$\begin{array}{c}\text { Hexa } \\
(2 \mathrm{~g} / \mathrm{l})+\mathrm{KI} \\
(0 \mathrm{~g} / \mathrm{l})\end{array}$ \\
0,844
\end{tabular}} & \multirow{2}{*}{\begin{tabular}{|c|c}
$\begin{array}{c}\text { Hexa } \\
(2 \mathrm{~g} / \mathrm{l})+\mathrm{KI} \\
(0,5 \mathrm{~g} / \mathrm{l})\end{array}$ \\
0,809
\end{tabular}} & \multirow{2}{*}{$\begin{array}{c}\begin{array}{c}\text { Hexa } \\
(2 \mathrm{~g} / \mathrm{l})+\mathrm{KI} \\
(1 \mathrm{~g} / \mathrm{l})\end{array} \\
0,751\end{array}$} & \multirow{2}{*}{$\begin{array}{c}\text { Hexa } \\
(2 \mathrm{~g} / \mathrm{l})+\mathrm{K} \\
(2 \mathrm{~g} / \mathrm{l}) \\
0,688\end{array}$} \\
\hline \multirow{3}{*}{$\begin{array}{c}\text { Condensate }: \text { nước }= \\
0: 100(\%)\end{array}$} & 4 giờ & & & & & \\
\hline & 8 giờ & 0,729 & 0,590 & 0,552 & 0,510 & 0,463 \\
\hline & 24 giờ & 0,542 & 0,402 & 0,368 & 0,331 & 0,310 \\
\hline \multirow{3}{*}{$\begin{array}{c}\text { Condensate }: \text { nước }= \\
50: 50(\%)\end{array}$} & 4 giờ & 0,846 & 0,704 & 0,646 & 0,606 & 0,552 \\
\hline & 8 giờ & 0,657 & 0,511 & 0,471 & 0,432 & 0,393 \\
\hline & 24 giờ & 0,463 & 0,336 & 0,306 & 0,280 & 0,255 \\
\hline \multirow{3}{*}{$\begin{array}{c}\text { Condensate }: \text { nước }= \\
70: 30(\%)\end{array}$} & 4 giờ & 0,730 & 0,560 & 0,522 & 0,514 & 0,491 \\
\hline & 8 giờ & 0,546 & 0,408 & 0,359 & 0,330 & 0,290 \\
\hline & 24 giờ & 0,405 & 0,278 & 0,252 & 0,228 & 0,200 \\
\hline \multirow{3}{*}{$\begin{array}{c}\text { Condensate }: \text { nước }= \\
90: 10(\%)\end{array}$} & 4 giờ & 0,428 & 0,339 & 0,270 & 0,248 & 0,214 \\
\hline & 8 giờ & 0,346 & 0,237 & 0,178 & 0,153 & 0,131 \\
\hline & 24 giờ & 0,248 & 0,137 & 0,099 & 0,080 & 0,074 \\
\hline
\end{tabular}


Bảng 3 cho thấy, khi có mặt chất ức chế "Hexa $(2 \mathrm{~g} / \mathrm{l})+\mathrm{KI}$ " trong các dung dịch nước và nhũ tương "condensate/nước", tốc độ ăn mòn thép giảm dần theo chiều tăng của thời gian ngâm mẫu, theo chiều giảm của hàm lượng nước trong các nhũ tương và theo chiều tăng của nồng độ KI.

So sánh số liệu trong các bảng $(3,4)$ với số liệu trong các bảng $(1,2)$ sau 4 giờ ngâm mẫu, ta còn thấy:

- Ở cùng nhiệt độ $30{ }^{\circ} \mathrm{C}$, tốc độ ăn mòn thép trong nước đã loại khí oxy $(0,42 \mathrm{~mm} / \mathrm{năm})$ thấp hơn 2 lần so với trong nước có khí oxy hòa tan $(0,98 \mathrm{~mm} / \mathrm{năm})$;

- Ở cùng nhiệt độ $30^{\circ} \mathrm{C}$, tốc độ ăn mòn thép trong nước đã loại oxy và có "Hexa $(2 \mathrm{~g} / \mathrm{l})+$ KI $(2 \mathrm{~g} / \mathrm{l})$ " là $0,19 \mathrm{~mm} / \mathrm{năm}$, thấp hơn gần 4 lần so với trong nước có oxy hòa tan $(0,69$ $\mathrm{mm} /$ năm).

Nói cách khác, ở nhiệt độ $30{ }^{\circ} \mathrm{C}$, chất ức chế "Hexa $(2 \mathrm{~g} / \mathrm{l})+\mathrm{KI}(2 \mathrm{~g} / \mathrm{l})$ " thể hiện hiệu quả bảo vệ thép trong nước không oxy $(54 \%)$ cao hơn khoảng 2 lần so với trong nước có oxy hòa $\tan (30 \%)$.

Bảng 4 cho thấy, trong các dung dịch nước và nhũ tương "condensate/nước" thông khí có mặt "Hexa $(2 \mathrm{~g} / \mathrm{l})+\mathrm{KI}(0 ; 0,5 ; 1 ; 2 \mathrm{~g} / \mathrm{l})$ ", hiệu quả ức chế ăn mòn thép tăng dần theo chiều tăng của thời gian ngâm mẫu, theo chiều giảm của hàm lượng nước, và theo chiều tăng của nồng độ KI . Như vậy, trong tất cả các dung dịch nước hoặc nhũ tương"condensate/nước" có khí oxy hòa tan ở $30^{\circ} \mathrm{C}$, hiệu quả bảo vệ thép luôn đạt giá trị cực đại ở nồng độ "Hexa $(2 \mathrm{~g} / \mathrm{l})+\mathrm{KI}$ ( $2 \mathrm{~g} / \mathrm{l})$ "'. Trong dung dịch nước không có khí oxy hòa tan, hiệu quả bảo vệ thép cũng luôn đạt giá trị cực đại ở nồng độ "Hexa $(2 \mathrm{~g} / \mathrm{l})+\mathrm{KI}(2 \mathrm{~g} / \mathrm{l})$ " trên khoảng rộng của nhiệt độ, từ $30^{\circ} \mathrm{C}$ đến $140{ }^{\circ} \mathrm{C}$.

Bảng 4. Hiệu quả bảo vệ thép của chất ức chế "Hexa $(2 \mathrm{~g} / \mathrm{l})+\mathrm{KI}(0 ; 0,5 ; 1 ; 2 \mathrm{~g} / \mathrm{l})$ " phụ thuộc vào nồng độ $\mathrm{KI}$, vào thời gian ngâm mẫu (giờ) và vào hàm lượng nước $(\%)$ trong các nhũ tương "condensate / nước" thông khí, ở nhiệt độ phòng.

\begin{tabular}{|c|c|c|c|c|c|}
\hline \multirow{2}{*}{\multicolumn{2}{|c|}{$\begin{array}{c}\text { Dung dịch “condensate/nước" (\%) và thời } \\
\text { gian ngâm mẫu (giờ) }\end{array}$}} & \multicolumn{4}{|c|}{ Hiệu quả bảo vệ thép (\%) } \\
\hline & & $\begin{array}{c}\text { Hexa } \\
(2 \mathrm{~g} / \mathrm{l})+\mathrm{Kl}\end{array}$ & $\begin{array}{c}\text { Hexa } \\
(2 \mathrm{~g} / \mathrm{l})+\mathrm{KI}\end{array}$ & Hexa $(2 \mathrm{~g} / \mathrm{l})$ & $\operatorname{Hexa}(2 \mathrm{~g} / \mathrm{l})$ \\
\hline \multirow{3}{*}{$\begin{array}{c}\text { Condensate }: \text { nước }= \\
0: 100(\%)\end{array}$} & 4 giờ & $14,25 \%$ & 17,79 & 23,63 & $30,07 \%$ \\
\hline & 8 giờ & 18,96 & 24,25 & 29,99 & 36,50 \\
\hline & 24 giờ & 25,74 & 32,00 & 38,90 & 42,69 \\
\hline \multirow{3}{*}{$\begin{array}{c}\text { Condensate }: \text { nước }= \\
50: 50(\%)\end{array}$} & 4 giờ & 16,87 & 23,66 & 28,38 & 34,73 \\
\hline & 8 giờ & 22,24 & 28,33 & 34,32 & 40,20 \\
\hline & 24 giờ & 27,47 & 33,85 & 39,51 & 44,97 \\
\hline \multirow{3}{*}{$\begin{array}{c}\text { Condensate }: \text { nước }= \\
70: 30(\%)\end{array}$} & 4 giờ & 17,84 & 28,45 & 29,54 & 32,78 \\
\hline & 8 giờ & 25,26 & 34,25 & 39,42 & 46,76 \\
\hline & 24 giờ & 31,23 & 37,82 & 43,77 & 50,59 \\
\hline \multirow{3}{*}{$\begin{array}{c}\text { Condensate }: \text { nước }= \\
90: 10(\%)\end{array}$} & 4 giờ & 20,89 & 36,96 & 42,23 & 50,06 \\
\hline & 8 giờ & 31,48 & 48,61 & 55,83 & 62,22 \\
\hline & 24 giờ & 44,90 & 60,08 & 67,68 & 70,24 \\
\hline
\end{tabular}




\subsection{Kết quả thí nghiệm trong nước, nhũ tương “condensate/nước" thông khí, ở nhiệt độ phòng bằng các phương pháp điện hóa}

Kết quả bảng 5 cho thấy:

- Trong nước, cũng như trong các nhũ tương "Condensate/nước", điện thế ăn mòn thép dịch chuyển dần về phía giá trị âm hơn theo chiều tăng của nồng độ KI.

- Độ dốc Tafel catot $\left(\beta_{c}\right)$ luôn cao hơn nhiều so với độ dốc Tafel anot $\left(\beta_{a}\right)$, chứng tỏ phản ứng catot xảy ra khó khăn hơn nhiều so với phản ứng anot.

Bảng 6 cho thấy, theo chiều tăng của nồng độ KI trong hỗn hợp "Hexa $(2 \mathrm{~g} / \mathrm{l})+\mathrm{KI}$ " và theo chiều giảm của hàm lượng nước trong các nhũ tương "Condensate/nước", điện thế ăn mòn thép dịch chuyển dần về phía có giá trị âm hơn, điện trở phân cực $\left(\mathrm{R}_{\mathrm{p}}\right)$ tăng dần, dần đến sự giảm dần mật độ dòng ăn mòn thép (i) và tốc độ ăn mòn thép $(\mathrm{V})$.

- Độ dốc Tafel anot $\left(\beta_{\mathrm{a}}\right)$ và catot $\left(\beta_{\mathrm{c}}\right)$ khi có mặt Hexa $(2 \mathrm{~g} / \mathrm{l})$, đều tăng lên theo chiều giảm của hàm lượng nước trong các nhũ tương "Condensate/nước" và theo chiều tăng của nồng độ KI trong hỗn hợp "Hexa $(2 \mathrm{~g} / \mathrm{l})+\mathrm{KI}$ ". Điều đó cho thấy "Hexa $(2 \mathrm{~g} / \mathrm{l})+\mathrm{KI}$ " đã ức chế cả phản ứng anot và phản ứng catot của sự ăn mòn điện hóa thép.

- Kết quả là, tốc độ ăn mòn thép trong các dung dịch đều giảm rõ rệt khi có mặt Hexa (2 $\mathrm{g} / \mathrm{l})$, giảm dần theo chiều tăng của nồng độ $\mathrm{KI}$ trong hốn hợp "Hexa $(2 \mathrm{~g} / \mathrm{l})+\mathrm{KI}$ " và theo chiều giảm của hàm lượng nước trong các nhũ tương.

Bảng 5. Kết quả đo các đường cong phân cực trong nước, trong nhũ tương "Condensate/nước", thông khí, ở nhiệt độ phòng. [Điện thế ăn mòn thép $\left(\mathrm{E}_{\mathrm{cor}}\right)$; độ dốc Tafel anot $\left(\beta_{\mathrm{a}}\right)$, catot $\left(\beta_{\mathrm{c}}\right)$; mật độ dòng ăn mòn thép (i) và tốc độ ăn mòn thép (V)].

\begin{tabular}{|c|c|c|c|c|c|c|}
\hline $\begin{array}{l}\text { Dung dịch } \\
\text { condensate/nư } \\
\text { ớc" }(\%)\end{array}$ & $\begin{array}{l}\text { Chất ức chế "Hexa } \\
\qquad(2 \mathrm{~g} / \mathrm{l})+\mathrm{KI} \text { " }\end{array}$ & $-E_{\text {corr }}(V)$ & $\beta_{\mathrm{a}}(\mathrm{mV})$ & $-\beta_{\mathrm{c}}(\mathrm{mV})$ & $\begin{array}{c}\text { i. } 10^{5} \\
\left(\mathrm{~mA} / \mathrm{cm}^{2}\right)\end{array}$ & $\begin{array}{c}\mathrm{V} \\
(\mathrm{mm} / \mathrm{năm})\end{array}$ \\
\hline \multirow{5}{*}{$\begin{array}{c}\text { Condensate : } \\
\text { nước }= \\
0: 100(\%)\end{array}$} & 0 & 0,397 & 86,6 & 587,7 & 10,880 & 1,265 \\
\hline & $\operatorname{Hexa}(2 \mathrm{~g} / \mathrm{l})+\mathrm{KI}(0 \mathrm{~g} / \mathrm{l})$ & 0,407 & 88,8 & 595,8 & 9,484 & 1,103 \\
\hline & $\begin{array}{l}\operatorname{Hexa}(2 \mathrm{~g} / \mathrm{l})+\mathrm{KI}(0,5 \\
\mathrm{g} / \mathrm{l})\end{array}$ & 0,411 & 89,9 & 596,7 & 9,236 & 1,085 \\
\hline & $\operatorname{Hexa}(2 \mathrm{~g} / \mathrm{l})+\mathrm{KI}(1 \mathrm{~g} / \mathrm{l})$ & 0,412 & 90,9 & 598,8 & 9,210 & 1,067 \\
\hline & $\operatorname{Hexa}(2 \mathrm{~g} / \mathrm{l})+\mathrm{KI}(2 \mathrm{~g} / \mathrm{l})$ & 0,414 & 104,2 & 699,6 & 8,461 & 0,984 \\
\hline \multirow{5}{*}{$\begin{array}{l}\text { Condensate : } \\
\text { nước = } \\
50: 50(\%)\end{array}$} & 0 & 0,381 & 85,1 & 652,7 & 10,456 & 1,216 \\
\hline & $\operatorname{Hexa}(2 \mathrm{~g} / \mathrm{l})+\mathrm{KI}(0 \mathrm{~g} / \mathrm{l})$ & 0,406 & 89,1 & 676,2 & 8,953 & 1,041 \\
\hline & $\begin{array}{l}\text { Hexa }(2 \mathrm{~g} / \mathrm{l})+\mathrm{KI}(0,5 \\
\mathrm{g} / \mathrm{l})\end{array}$ & 0,412 & 92,3 & 679,3 & 8,808 & 1,024 \\
\hline & $\operatorname{Hexa}(2 \mathrm{~g} / \mathrm{l})+\mathrm{KI}(1 \mathrm{~g} / \mathrm{l})$ & 0,412 & 95,1 & 682,5 & 8,182 & 0,952 \\
\hline & $\operatorname{Hexa}(2 \mathrm{~g} / \mathrm{l})+\mathrm{KI}(2 \mathrm{~g} / \mathrm{l})$ & 0,413 & 95,6 & 687,7 & 7,170 & 0,834 \\
\hline \multirow{5}{*}{$\begin{array}{c}\text { Condensate: } \\
\text { nước }= \\
70: 30(\%)\end{array}$} & 0 & 0,397 & 102,2 & 724,7 & 9,944 & 1,157 \\
\hline & $\operatorname{Hexa}(2 \mathrm{~g} / \mathrm{l})+\mathrm{KI}(0 \mathrm{~g} / \mathrm{l})$ & 0,404 & 102,6 & 874,1 & 7,077 & 0,823 \\
\hline & $\begin{array}{l}\operatorname{Hexa}(2 \mathrm{~g} / \mathrm{l})+\mathrm{KI}(0,5 \\
\mathrm{g} / \mathrm{l})\end{array}$ & 0,416 & 104,8 & 880,9 & 5,968 & 0,694 \\
\hline & $\operatorname{Hexa}(2 \mathrm{~g} / \mathrm{l})+\mathrm{KI}(1 \mathrm{~g} / \mathrm{l})$ & 0,417 & 106,6 & 903,1 & 5,343 & 0,621 \\
\hline & $\operatorname{Hexa}(2 \mathrm{~g} / \mathrm{l})+\mathrm{KI}(2 \mathrm{~g} / \mathrm{l})$ & 0,419 & 111,4 & 914,1 & 5,239 & 0,609 \\
\hline $90: 10(\%)$ & \multicolumn{6}{|c|}{ Không đo được vì độ dẫn điện của dung dịch quá thấp } \\
\hline 100:0 (\%) & \multicolumn{6}{|c|}{ Không đo được vì độ dẫn điện của dung dịch quá thấp } \\
\hline
\end{tabular}


Ảnh hưởng hỗ trợ của các ion iodua đến sự ức chế ăn mòn thép cacbon ...

Bảng 6. Điện thế ăn mòn $\left(\mathrm{E}_{\mathrm{cor}}\right)$; điện trở phân cực $\left(\mathrm{R}_{\mathrm{p}}\right)$; mật độ dòng ăn mòn thép (i), tốc độ ăn mòn thép (V) nhận được bằng phương pháp đo điện trở phân cực trong nước và nhũ tương "condensate /nước" thông khí, ở nhiệt độ phòng.

\begin{tabular}{|c|c|c|c|c|c|}
\hline $\begin{array}{l}\text { Dung dịch } \\
\text { "Condensate: } \\
\text { Nước" }(\%)\end{array}$ & Chất ức chế & $\begin{array}{c}-\mathrm{E}_{\mathrm{cor}} \\
(\mathrm{V})\end{array}$ & $\begin{array}{c}\mathrm{R}_{\mathrm{p}} \\
\left(\Omega . \mathrm{cm}^{2}\right)\end{array}$ & $\begin{array}{c}\mathrm{i} .10^{5} \\
\left(\mathrm{~mA} / \mathrm{cm}^{2}\right)\end{array}$ & $\begin{array}{c}\mathrm{V} \\
(\mathrm{mm} / \mathrm{năm})\end{array}$ \\
\hline \multirow{5}{*}{$0: 100(\%)$} & 0 & 0,413 & 263,4 & 9,871 & 1,148 \\
\hline & Hexa $(2 \mathrm{~g} / \mathrm{l})$ & 0,422 & 273,5 & 9,505 & 1,106 \\
\hline & Hexa $(2 \mathrm{~g} / \mathrm{l})+\mathrm{KI}(0,5 \mathrm{~g} / \mathrm{l})$ & 0,423 & 277,0 & 9,387 & 1,092 \\
\hline & $\operatorname{Hexa}(2 \mathrm{~g} / \mathrm{l})+\mathrm{KI}(1 \mathrm{~g} / \mathrm{l})$ & 0,423 & 286,6 & 9,073 & 1,055 \\
\hline & $\operatorname{Hexa}(2 \mathrm{~g} / \mathrm{l})+\mathrm{KI}(2 \mathrm{~g} / \mathrm{l})$ & 0,425 & 298,9 & 8,698 & 1,012 \\
\hline \multirow{5}{*}{$50: 50(\%)$} & 0 & 0,390 & 283,1 & 9,184 & 1,068 \\
\hline & Hexa (2 g/l) & 0,418 & 345,3 & 7,531 & 0,876 \\
\hline & $\operatorname{Hexa}(2 \mathrm{~g} / \mathrm{l})+\mathrm{KI}(0,5 \mathrm{~g} / \mathrm{l})$ & 0,426 & 350,5 & 7,418 & 0,863 \\
\hline & $\operatorname{Hexa}(2 \mathrm{~g} / \mathrm{l})+\mathrm{KI}(1 \mathrm{~g} / \mathrm{l})$ & 0,427 & 372,5 & 6,979 & 0,812 \\
\hline & $\operatorname{Hexa}(2 \mathrm{~g} / \mathrm{l})+\mathrm{KI}(2 \mathrm{~g} / \mathrm{l})$ & 0,428 & 387,6 & 6,708 & 0,780 \\
\hline \multirow{5}{*}{$70: 30(\%)$} & 0 & 0,422 & 335,4 & 7,315 & 0,851 \\
\hline & $\operatorname{Hexa}(2 \mathrm{~g} / \mathrm{l})$ & 0,425 & 536,5 & 4,846 & 0,564 \\
\hline & $\operatorname{Hexa}(2 \mathrm{~g} / \mathrm{l})+\mathrm{KI}(0,5 \mathrm{~g} / \mathrm{l})$ & 0,431 & 587,4 & 4,426 & 0,515 \\
\hline & $\operatorname{Hexa}(2 \mathrm{~g} / \mathrm{l})+\mathrm{KI}(1 \mathrm{~g} / \mathrm{l})$ & 0,432 & 602,1 & 4,318 & 0,502 \\
\hline & $\operatorname{Hexa}(2 \mathrm{~g} / \mathrm{l})+\mathrm{KI}(2 \mathrm{~g} / \mathrm{l})$ & 0,432 & 679,2 & 3,828 & 0,445 \\
\hline $90: 10(\%)$ & \multicolumn{5}{|c|}{ Không đo được vì độ dẫn điện của dung dịch quá thấp } \\
\hline 100:0 (\%) & \multicolumn{5}{|c|}{ Không đo được vì độ dẫn điện của dung dịch quá thấp } \\
\hline
\end{tabular}

Bảng 7. Điện trở phân cực $\left(\mathrm{R}_{\mathrm{p}}\right)$, điện dung lớp điện tích kép $(\mathrm{C})$ trên thép nhận được bằng phương pháp đo tổng trở điện hóa trong các dung dịch nước, nhũ tương “condensate/nước” thông khí, ở nhiệt độ phòng.

\begin{tabular}{|c|c|c|c|}
\hline $\begin{array}{c}\text { Dung dịch "Condensate/ } \\
\text { Nước" }(\%)\end{array}$ & Chất ức chế & $\mathrm{R}_{\mathrm{p}}\left(\Omega \cdot \mathrm{cm}^{2}\right)$ & $\mathrm{C} \cdot 10^{5}\left(\mathrm{~F} / \mathrm{cm}^{2}\right)$ \\
\hline \multirow{4}{*}{$0: 100(\%)$} & 0 & 73,4 & 26,445 \\
\cline { 2 - 4 } & Hexa $(2 \mathrm{~g} / \mathrm{l})$ & 75,6 & 23,445 \\
\cline { 2 - 4 } & Hexa $(2 \mathrm{~g} / \mathrm{l})+\mathrm{KI}(0,5 \mathrm{~g} / \mathrm{l})$ & 82,4 & 17,347 \\
\cline { 2 - 4 } & Hexa $(2 \mathrm{~g} / \mathrm{l})+\mathrm{KI}(1 \mathrm{~g} / \mathrm{l})$ & 85,2 & 16,668 \\
\cline { 2 - 4 } & Hexa $(2 \mathrm{~g} / \mathrm{l})+\mathrm{KI}(2 \mathrm{~g} / \mathrm{l})$ & 88,4 & 14,771 \\
\hline \multirow{5}{*}{$50: 50(\%)$} & 0 & 94,8 & 8,580 \\
\cline { 2 - 4 } & Hexa $(2 \mathrm{~g} / \mathrm{l})$ & 98,9 & 8,257 \\
\cline { 2 - 4 } & Hexa $(2 \mathrm{~g} / \mathrm{l})+\mathrm{KI}(0,5 \mathrm{~g} / \mathrm{l})$ & 101,6 & 7,867 \\
\cline { 2 - 4 } & Hexa $(2 \mathrm{~g} / \mathrm{l})+\mathrm{KI}(1 \mathrm{~g} / \mathrm{l})$ & 112,8 & 5,719 \\
\cline { 2 - 4 } & Hexa $(2 \mathrm{~g} / \mathrm{l})+\mathrm{KI}(2 \mathrm{~g} / \mathrm{l})$ & 118,9 & 5,374 \\
\hline
\end{tabular}




\begin{tabular}{|c|c|c|c|}
\hline \multirow{3}{*}{$70: 30(\%)$} & 0 & 109,9 & 2,126 \\
\cline { 2 - 4 } & Hexa $(2 \mathrm{~g} / \mathrm{l})$ & 123,7 & 1,932 \\
\cline { 2 - 4 } & Hexa $(2 \mathrm{~g} / \mathrm{l})+\mathrm{KI}(0,5 \mathrm{~g} / \mathrm{l})$ & 176,8 & 1,768 \\
\cline { 2 - 4 } & Hexa $(2 \mathrm{~g} / \mathrm{l})+\mathrm{KI}(1 \mathrm{~g} / \mathrm{l})$ & 193,2 & 1,237 \\
\cline { 2 - 4 } & Hexa $(2 \mathrm{~g} / \mathrm{l})+\mathrm{KI}(2 \mathrm{~g} / \mathrm{l})$ & 212,6 & 1,099 \\
\hline $90: 10(\%)$ & \multicolumn{2}{|c|}{ Không đo được vì độ dẫn điện của dung dịch quá thấp } \\
\hline $100: 0(\%)$ & \multicolumn{2}{|c|}{ Không đo được vì độ dẫn điện của dung dịch quá thấp } \\
\hline
\end{tabular}

Như thấy trong bảng 7 , theo chiều giảm của hàm lượng nước trong nhũ tương "condensate/nước" (từ $100 \%$ xuống $30 \%$ nước), cũng như theo chiều tăng của nồng độ KI trong hỗn hợp "Hexa $(2 \mathrm{~g} / \mathrm{l})+\mathrm{KI})$ " (từ 0 đến $2 \mathrm{~g} / \mathrm{l} \mathrm{KI})$, thì giá trị điện trở phân cực $\left(\mathrm{R}_{\mathrm{p}}\right)$ tăng dần, giá trị điện dung của lớp điện tích kép $(\mathrm{C})$ trên thép giảm dần.

\section{Giải thích các kết quả thực nghiệm}

Condensate là các hydrocarbon mạch thẳng không dẫn điện và không ăn mòn thép. Dung dịch nước lẫn trong condensate có tính axit và có độ dẫn điện cao đã gây ra sự ăn mòn điện hóa thép trong các dung dịch nước và nhũ tương "condensate/nước" theo các phản ứng sau:

* Trong dung dịch axit $(\mathrm{pH}<7)$, không có khí oxy hòa tan:

Trên bề mặt điện cực anot, nguyên tử sắt bị oxy hóa (thép bị ăn mòn):

$$
\mathrm{Fe} \rightarrow \mathrm{Fe}^{2+}+2 \mathrm{e}^{-}
$$

Trên bề mặt điện cực catot, ion hydro bị khử:

$$
2 \mathrm{H}^{+}+2 \mathrm{e}^{-} \rightarrow \mathrm{H}_{2}
$$

* Trong dung dịch axit $(\mathrm{pH}<7)$, có khí oxy hòa tan:

Trên điện cực anot:

$$
\mathrm{Fe} \rightarrow \mathrm{Fe}^{2+}+2 \mathrm{e}^{-}
$$

Trên điện cực catot:

$$
\begin{aligned}
& 2 \mathrm{H}^{+}+2 \mathrm{e}^{-} \rightarrow \mathrm{H}_{2} \\
& \mathrm{O}_{2}+4 \mathrm{H}^{+}+4 \mathrm{e}^{-} \rightarrow 2 \mathrm{H}_{2} \mathrm{O}
\end{aligned}
$$

Phản ứng anot (3) xảy ra dễ dàng, thể hiện ở độ dốc $\left(\beta_{a}\right)$ thấp của các đường cong phân cực anot; phản ứng catot (4) (5) xảy ra khó khăn hơn nhiều, thể hiện ở độ dốc cao $\left(\beta_{c}\right)$ của các đường cong phân cực catot (bảng 5).

- Khí oxy hòa tan đã khử phân cực catot theo phản ứng (5), làm cho tốc độ ăn mòn thép trong nước có mặt oxy ở nhiệt độ phòng (bảng 3) cao hơn 2 lần so với trong nước đã loại khí oxy ở cùng nhiệt độ (bảng 1).

- Tốc độ ăn mòn thép trong các nhũ tương "condensate/nước" đã giảm dần theo chiều giảm của hàm lượng nước (bảng $3,5,6$ ), vì hai nguyên nhân sau: Một là, các phản ứng anot và catot của sự ăn mòn điện hóa thép đều xảy ra khó khăn hơn khi hàm lượng nước giảm, do điện trở của dung dịch tăng. Hai là, hàm lượng nước giảm dần, đồng nghĩa với sự tăng dần nồng độ của chất ức chế Hexa và chất phụ trợ KI trong các dung dịch, vì Hexa và KI chỉ hòa tan được trong nước và không hòa tan trong condensate. 
Hexa có công thức phân tử $\left(\mathrm{CH}_{2}\right)_{6} \mathrm{~N}_{4}$, là hợp chất hữu cơ dị vòng.

Khi cho $2 \mathrm{~g} / \mathrm{l}$ Hexa vào trong các dung dịch nước và nhũ tương "Condensate/nước"có tính axit, Hexa có thể bị proton hóa thành cation tích điện dương dưới dạng $\left[\left(\mathrm{CH}_{2}\right)_{6} \mathrm{~N}_{4} \mathrm{H}_{4}\right]^{4+}$, nhờ tương tác giữa 4 cặp điện tử đơn độc của 4 nguyên tử ni tơ trong 1 phân tử Hexa với các cation hydro $\mathrm{H}^{+}$:

$$
\left(\mathrm{CH}_{2}\right)_{6} \mathrm{~N}_{4}+4 \mathrm{H}^{+} \leftrightarrow\left[\left(\mathrm{CH}_{2}\right)_{6} \mathrm{~N}_{4} \mathrm{H}_{4}\right]^{4+}
$$

Các cation $\left[\left(\mathrm{CH}_{2}\right)_{6} \mathrm{~N}_{4} \mathrm{H}_{4}\right]^{4+}$ hấp phụ lên bề mặt thép tích điện âm, tạo thành lớp hàng rào đơn tích điện dương.

Khi có mặt các anion $\mathrm{I}^{-}$tích điện âm trong dung dịch (do phản ứng phân ly $\mathrm{KI} \rightarrow \mathrm{K}^{+}+\mathrm{I}^{-}$), các cation tích điện dương $\left[\left(\mathrm{CH}_{2}\right)_{6} \mathrm{~N}_{4} \mathrm{H}_{4}\right]^{4+}$ sẽ tương tác tĩnh điện với các anion I' tạo thành lớp hàng rào kép tích điện âm $\left\{\left[\left(\mathrm{CH}_{2}\right)_{6} \mathrm{~N}_{4} \mathrm{H}_{4}\right]^{4+}-\mathrm{I}^{-}\right\}$trên bề mặt thép. Lớp hàng rào kép tích điện âm này gây ra các hệ quả sau, theo chiều tăng của nồng độ Hexa và của nồng độ KI trong hỗn hợp "Hexa $(2 \mathrm{~g} / \mathrm{l})+\mathrm{KI}(0 ; 0,5 ; 1 ; 2 \mathrm{~g} / \mathrm{l})$ ":

- Làm cho điện thế ăn mòn thép $\left(\mathrm{E}_{\mathrm{cor}}\right)$ dịch chuyển dần về phía có giá trị âm hơn (bảng 5,6 );

- Làm tăng độ dốc Tafel anot $\left(\beta_{\mathrm{a}}\right)$ và catot $\left(\beta_{\mathrm{c}}\right)$, nghĩa là ức chế các phản ứng anot và catot của sự ăn mòn điện hóa thép (bảng 5,6 );

- Làm tăng điện trở phân cực $\left(\mathrm{R}_{\mathrm{p}}\right)$ và làm giảm điện dung $(\mathrm{C})$ của lớp điện tích kép (bảng 7);

- Kết quả là, làm giảm tốc độ ăn mòn thép trong các dung dịch nước và nhũ tương “condensate /nước" với hàm lượng nước giảm dần (bảng 5,6 ).

Trong dung dịch nước, hiệu quả bảo vệ thép của hỗn hợp "Hexa $(2 \mathrm{~g} / \mathrm{l})+\mathrm{KI}(0 ; 0,5 ; 1$; $2 \mathrm{~g} / \mathrm{l}$ )" có một cực đại ở $100{ }^{\circ} \mathrm{C}$ (bảng 2). Điều đó có thể giải thích là do ở nhiệt độ cao $140{ }^{\circ} \mathrm{C}$, chuyển động hỗn loạn quá nhanh của các phân tử nước và các ion trong dung dịch đã phần nào phá vỡ mức độ dày đặc của lớp hàng rào kép trên bề mặt thép.

\section{KẾT LUẬN}

Trong nước có tính axit đã loại khí oxy, tốc độ ăn mòn thép bị tăng dần theo chiều tăng của nhiệt độ, từ $30^{\circ} \mathrm{C}$ lên $140{ }^{\circ} \mathrm{C}$;

Tốc độ ăn mòn thép trong nước không hoặc có oxy, cũng như trong các nhũ tương "condensate/nước", được giảm rõ rệt khi có mặt chất ức chế "Hexa $(2 \mathrm{~g} / \mathrm{l})$ " và giảm dần theo chiều tăng của nồng độ KI trong hồn hợp "Hexa $(2 \mathrm{~g} / \mathrm{l})+\mathrm{KI}(0 ; 0,5 ; 1 ; 2 \mathrm{~g} / \mathrm{l})$ ".

Tốc độ ăn mòn thép càng thấp, nếu hàm lượng nước trong các nhũ tương "condensate/nước" càng thấp.

Hiệu quả bảo vệ thép bởi hỗn hợp "Hexa $(2 \mathrm{~g} / \mathrm{l})+\mathrm{KI}(2 \mathrm{~g} / \mathrm{l})$ " trong nước đã loại khí oxy cao hơn khoảng 2 lần so với trong nước có oxy ở cùng nhiệt độ.

Cơ chế ức chế ăn mòn thép của hỗn hợp "Hexa $(2$ g/l $)+$ KI" được giải thích là do có sự tạo thành lớp hấp phụ kép trên bề mặt thép trong nước cũng như trong các nhũ tương "condensate/nước".

\section{TÀI LIỆU THAM KHẢO}

1. ASTM G1 - 90: Standard practice for preparing, cleaning, and evaluating corrosion test specimens. 
2. Volovitch P., Gazizzullin I., Ruel F., Ogle K. - An atomic emission spectroelectrochemical study of corrosion inhibition: The effect of hexamethylenetetramine on the reaction of mild steel in $\mathrm{HCl}$, Corrosion Science $\mathbf{5 3}$ (2001) 1362-1368.

3. Brolo A. G., Temperini M. L. A., and Agostinho S. M. L. - Effect of hexamethylenetetramine as a corrosion inhibitor for copper in bromide medium, J. Electroanal. Chem. 335 (1992) 83-92.

4. Eduok U. M., Umoren S. A., Udoh A. P. - Synergistic inhibition effects between leaves and stem extracts of Sida acuta and iodide ion for mild steel corrosion in $1 \mathrm{M} \mathrm{H}_{2} \mathrm{SO}_{4}$ solutions, Arabian Journal of Chemistry 5 (2012) 325-337.

5. Oribite-Okorosaye K., Jack I. R., Ochei M., Akaranta O. - Synergistic Effect of Potassium Iodide on Corrosion Inhibition of Mild Steel in $\mathrm{HCl}$ Medium by Extracts of NypaFruticans' Wurmb , J. Appl. Sci. Environ. Manage. June 11 (2) (2007) 27-31.

6. Larabi L., Harek Y., Traisnel M., and Mansri A. - Synergistic influence of poly(4vinylpyridine) and potassium iodide on inhibition of corrosion of mild steel in $1 \mathrm{M} \mathrm{HCl}$, Journal of Applied Electrochemistry 34 (2004) 833-839.

7. Sadaf Khan, Quraishi M. A. - Synergistic effect of potassium iodide on inhibitive performance of thiadiazoles during corrosion of mild steel in $20 \%$ sulfuric acid, The Arabian Journal for Science and Engineering 35 (1A) ( 2009) 71-82.

8. Shaju K. S., Joby Thomas K., Vinod P. Raphael, Aby Paul - Synergistic effect of KI on corrosion inhibition efficacy of a polynuclear schief base in sulfuric acid media, Department of Chemistry, St.Thomas' College (University of Calicut) Thrissur, Kerala, India, 2010.

9. Syed Azim S., Muralidharan S., Venkatakrishna Iyer S. - Studies on the influence of iodide ion on the synergistic inhibition of the corrosion of mild steel in an acidic solution, Journal of applied electrochemistry 25 (1995) 495-500.

10. Bentiss F., Bouani M., Mernari B., Traisnel M., and Lagrenee M. - Effect of iodide ions on corrosion inhibition of mild steel by 3, 5-bis (4-methylthiophenyl)-4H-1, 2, 4-triazole in sulfuric acid solution, Journal of Applied Electrochemistry 32 (2002) 671-678.

11. Umoren S. A., Solomon M. M., Udosoro I. I., Udoh A. P. - Synergistic and antagonistic effects between halide ions and carboxymethyl cellulose for the corrosion inhibition of mild steel in sulphuric acid solution. Springer Science Cellulose 17 (2010) 635-648.

12. Syed Azim S., Muralidharan S., Venkatakrishna Iyer S. - Studies on the influence of iodide ion on the synergistic inhibition of the corrosion of mild steel in an acidic solution, Journal of applied electrochemistry 25 (1995) 495-500.

13. Bentiss F., Bouani M., Mernari B., Traisnel M., and Lagrenee M. - Effect of iodide ions on corrosion inhibition of mild steel by 3, 5-bis (4-methylthiophenyl)-4H-1, 2, 4-triazole in sulfuric acid solution, Journal of Applied Electrochemistry 32 (2002) 671-678.

14. Umoren S. A., Solomon M. M., Udosoro I. I., Udoh A. P. - Synergistic and antagonistic effects between halide ions and carboxymethyl cellulose for the corrosion inhibition of mild steel in sulphuric acid solution, Springer Science Cellulose 17 (2010) 635-648.

15. ASTM G111 - 92: Standard guide for corrosion test in the high temperature or high pressure environment, or both. 
16. ASTM G5 - 94: Standard reference test method for making potentiostatic and potentiodynamic anodic polarization.

17. ASTM G59 - 91: Conducting Potentiodynamic Polarization Resistance Measurements.

18. ASTM G106 - 89: Standard practice for verification of algorithm and equipment for electrochemical impedance measurement.

\author{
ABSTRACT \\ SYNERGISTIC EFFECT OF IODIDE IONS ON THE CORROSION INHIBITION OF \\ CARBON STEEL BY HEXAMETHYLENETETRAMINE IN THE WATER AND \\ "CONDENSATE/WATER" EMULSIONS \\ Vu Dinh Huy ${ }^{*}$, Luu Hoang Tam, Le Van Thuan, Thai Nhi Hiep, Phan Thanh Binh \\ Ho Chi Minh City University of Technology, 268 Ly Thuong Kiet, Ho Chi Minh City \\ "Email: huyvvudinh@yahoo.com
}

The synergistic action caused by iodide ions $\left(\mathrm{I}^{-}\right)$on the corrosion inhibition of carbon steel in the water and "condensate/water" emulsions in the presence of Hexamethylenetetramine $\left(\mathrm{C}_{6} \mathrm{H}_{12} \mathrm{~N}_{4}\right)$ has been investigated using mass-loss measurements, potentiodynamic polarization, linear polarization and electrochemical impedance techniques. Hexamethylenetetramine (Hexa) inhibits the corrosion of carbon steel even at lower concentrations. The steel corrosion inhibition efficiency of Hexa is increased with increasing concentration of iodide ions and with decreased of water content in condensate/water" emulsions. The steel corrosion inhibition efficiency by "Hexa $(2 \mathrm{~g} / \mathrm{l})+\mathrm{KI}(0,0.5,1,2 \mathrm{~g} / \mathrm{l})$ " mixture is increased significantly with dissolved oxygen exclusion from water. The highest steel corrosion inhibition efficiency by "Hexa $(2 \mathrm{~g} / \mathrm{l})+\mathrm{KI}(0$, $0.5,1,2 \mathrm{~g} / \mathrm{l})$ " mixture in the water is observed at $100{ }^{\circ} \mathrm{C}$ in the temperature range from $30{ }^{\circ} \mathrm{C}$ to $140{ }^{\circ} \mathrm{C}$. The mechanism of corrosion inhibition by "Hexa $+\mathrm{KI}$ " mixture attributed to the formation of double layers adsorbed on the steel surface.

Keywords: iodide ions, hexamethylenetetramine, corrosion inhibition, carbon steel, water, "condensate/water" emulsions. 